\title{
Copy number analysis of survival motor neuron genes by multiplex ligation-dependent probe amplification
}

Chien-Hao Huang, $M S^{1}$, Yih-Yuan Chang, $M S^{1}$, Chung-Hsiung Chen, $B S^{1}$, Yen-Shi Kuo, $B S^{1}$, Wuh-Liang Hwu, $M D, P h D^{2}$, Tommy Gerdes, $M S^{3}$, and Tsang-Ming Ko, $M D, P h D^{1}$

\begin{abstract}
Purpose: To determine the copy number of survival motor genes using multiplex ligation-dependent probe amplification. Methods: Three hundred seventy-three subjects were recruited and divided into three groups. Group 1 included 310 subjects without a history of muscular atrophy, Group 2 consisted of 18 patients and 45 carriers of spinal muscular atrophy, and Group 3 included 20 subjects who were previously tested with denatured high-performance liquid chromatography. The copy number of survival motor neuron 1 and survival motor neuron 2 genes was determined with a commercially available multiplex ligation-dependent probe amplification kit. Results: Twenty-one genotypes of the survival motor neuron genes could be clearly defined in this series. The whole process of genotyping took $<48$ hours. In Group 1, 2:2 (survival motor neuron 1:survival motor neuron 2) was most common (52.90\%), followed by 2:1 (30.32\%); six (1.94\%) subjects were found to be carriers of 1:2 or 1:3. In Group 2 , all 18 patients had zero copies of the survival motor neuron 1 gene and variable copies of the survival motor neuron 2 gene. In Group 3, three subjects who had been told they were carriers of spinal muscular atrophy turned out to be noncarriers by multiplex ligation-dependent probe amplification. All 51 carriers from Groups 1 and 2 had one copy of the survival motor neuron 1 gene and one to four copies of the survival motor neuron 2 gene. Conclusion: Multiplex ligation-dependent probe amplification is a simple and efficient method for copy number analysis of survival motor neuron genes. It can be used to detect the homozygous and heterozygous survival motor neuron deletion of spinal muscular atrophy. Genet Med 2007:9(4):241-248.
\end{abstract}

Key Words: Spinal muscular atrophy, survival motor neuron 1 gene, survival motor neuron 2 gene, spinal muscular atrophy, survival motor neuron

Deletion of the survival motor neuron $(\mathrm{SMN})$ gene is the most common molecular basis of spinal muscular atrophy (SMA), which is a common autosomal recessive neuromuscular disease characterized by degeneration of the anterior horn cells of the spinal cord. The carrier rate of SMA varies from $2 \%$ to $3 \%$ in different populations. ${ }^{1-3}$ SMA is generally divided into three groups, based on age at onset and disease severity: 1 . Type I (infantile type, OMIM\# 253300). These patients usually develop muscle weakness at birth or before 6 months of age and die of respiratory failure within 2 years. 2. Type II (OMIM\# 253550). The onset is usually 18 months after birth. These patients can sit, but are never able to walk by themselves

\footnotetext{
From the ${ }^{1}$ Genephile Bioscience Laboratory, Ko's Obstetrics and Gynecology, Taipei, Taiwan; ${ }^{2}$ Department of Pediatrics, National Taiwan University, Taipei, Taiwan; and ${ }^{3}$ Department of Clinical Genetics, Rigshospitalet, Copenhagen, Denmark.

Dr. Tsang-Ming Ko, MD, PhD, 10 Lin-Sen South Road, Taipei, Taiwan; E-mail: tsangming@ gigigaga.com

The authors declare no conflict of interest.

Submitted for publication September 6, 2006.

Accepted for publication January 24, 2007.

DOI: 10.1097/GIM.0b013e31803d35bc
}

and can survive beyond 4 years of age. 3. Type III (OMIM\# 253400). These patients develop symptoms after 18 months and are able to walk, but often become wheelchair bound during youth or adulthood. ${ }^{4}$

Two highly homologous $S M N$ genes (SMN1 and SMN2) are located in the highly duplicated region of $5 \mathrm{q} 13$. SMN consists of nine exons (1, 2a, 2b, 3-8), with the stop codon present near the end of exon 7. The coding sequence of SMN2 differs from that of $S M N 1$ by a single nucleotide $(840 \mathrm{C}>\mathrm{T})$ in exon 7 . In exon 8, SMN2 differs from SMN1 by another single nucleotide $(G>A)$. Most of the SMN protein in a normal subject is derived from SMN1, and only a very small amount of SMN protein is derived from SMN2. Gene deletion or conversion is relatively common between $S M N 1$ and $S M N 2$, due to the sequence homology of the two SMN genes and their flanking regions. More than $95 \%$ of the SMA alleles of all three types of patients are due to deletion or gene conversion of SMN1, and about $5 \%$ of the diseased alleles are due to intragenic point mutations of SMN1. In patients with a total deletion of both copies of SMN1, the presence of SMN2 genes alleviates the disease severity. Compared with Type I patients, a higher proportion of Type III patients have four copies of SMN2.5,6 Many 
different methods for determining the copy number of SMN1 have been reported, including single-strand conformation polymorphism (SSCP), polymerase chain reaction (PCR)-restriction-fragment length polymorphism (RFLP), denatured high-performance liquid chromatography (DHPLC), and real-time quantitative PCR. ${ }^{7-14}$

In 2002, Schouten et al. ${ }^{15}$ reported a novel method for the relative quantification of up to 40 nucleic acid sequences in a single-tube, multiplex ligation-dependent probe amplification (MLPA). This method has been applied to detect deletion and duplication in many conditions, including trisomy syndromes, breast cancer, colon cancer, and Duchenne muscular dystrophy. ${ }^{16-22}$ Two reports have been published addressing SMA with MLPA. ${ }^{23,24}$ In this study, we used MLPA to analyze the copy number of the SMN1 and SMN2 genes in Taiwanese subjects and compared the MLPA results with those of DHPLC in 20 subjects.

\section{MATERIALS AND METHODS}

Three groups of subjects were recruited for this series. Group 1 consisted of 310 unrelated subjects without a history of muscular atrophy; Group 2 was composed of 63 subjects with 18 SMA patients and 45 carriers; and Group 3 comprised 20 subjects who had been screened with DHPLC at other laboratories, with 19 being told they were carriers of SMA. Informed consent to participate in the study was obtained from each subject. Among the 45 carriers in Group 2, 29 were parents of the patients, and 16 were ascertained through DHPLC or by repeated MLPA analysis.

DNA was extracted, using standard methods, from the peripheral blood of each subject. A spectrophotometer was used to determine the concentration of the DNA samples. Using a commercially available Salsa MLPA kit P021 (MRC-Holland), we genotyped the copy number of SMN1 and SMN2 in each subject. The kit contained 16 probes in the $5 \mathrm{q} 13$ region and another 22 control probes from other chromosomes. Details of probe sequences and gene loci are shown on the Web site (http://www.mrc-holland.com).

Among the 16 probes from the $5 \mathrm{q} 13$ region, eight were used to detect the copy numbers of SMN1 and/or SMN2: the SMN1-D01 probe for the copy number of $S M N 1$ exon 7; the SMN2-D01 probe for the copy number of SMN2 exon 7; the SMN1-D07 probe for the copy number of SMN1 exon 8; the SMN2-D07 probe for the copy number of SMN2 exon 8; and four probes, SMN1/2-D03, -D04, -D05, and -D06, for the copy number detection of exons $8,1,4,6$, respectively, for both SMN1 and SMN2.

MLPA analysis was performed according to the manufacturer's protocol (MRC Holland). Briefly, 50-500 ng of DNA was denatured $\left(98^{\circ} \mathrm{C}, 5\right.$ minutes) and hybridized with the probe set overnight at $60^{\circ} \mathrm{C}$, using the SALSA probe mix. Ligation was performed with ligase- 65 enzyme at $54^{\circ} \mathrm{C}$ for $15 \mathrm{~min}$ utes. The reactions were inactivated by incubation at $98^{\circ} \mathrm{C}$ for 15 minutes. PCR was performed with the specific SALSA FAM PCR primers for 35 cycles $\left(95^{\circ} \mathrm{C}\right.$ for 30 seconds; $60^{\circ} \mathrm{C}$ for 30 seconds; $72^{\circ} \mathrm{C}$ for 1 minute). The PCR products were analyzed in a capillary DNA sequencer (ABI PRISM 3100, Applied Biosystems, Darmstadt, Germany) with Genescan 3.7 Software (Applied Biosystems). One microliter of the PCR product was mixed with $0.5 \mu \mathrm{L}$ of Genescan-Rox 500 size standard and 8.5 $\mu \mathrm{L}$ of deionized formamide. The run time was 60 minutes using a $36-\mathrm{cm}$ capillary at $60^{\circ} \mathrm{C}$.

\section{Data analysis}

According to the length (bp, base pairs) of the PCR products, the 38 probes, with the $165 \mathrm{q} 13$ probes shown in boldface, were divided into four groups, namely group A (94-211 bp) (10 probes: 2q14, 12q14, CDH6-D01, 3q12, 17p11.2, 18q21.2, GTF2H2-D02, 7q21, RAD17-D02, and 10p15.1); group B (220-310 bp) (11 probes: GTF2H2-D03, 11q13, BIRC1-D02, 21q11, 18p11, SMN1-D01, SMN2-D01, 3q21, SMN1-D07, SMN2-D07, and 13q34); group C (319-391 bp) (nine probes: 8q24, GTF2H2-D01, 21q21.3, BIRC1-D01, 2p24, SMN1/2D03, 8p11.2, SMN1/2-D04, and 3p26); and group D (400-463 bp) (eight probes: SMN1/2-D05, 2p14, SMN1/2-D06, 8q24.11, 17q12, 13q34, N-cadherin-like-D01, and 12q24.13) (Fig. 1).

Data analysis was performed as reported by Gerdes et al. ${ }^{25}$ (the software is free and can be downloaded at www.chromosomelab.dk). Briefly, after automatic peak recognition and normalization, a probe ratio of each peak was computed by dividing the area by a corresponding reference mean peak area. The normalization was done separately for Groups A, B, C, and $\mathrm{D}$, but for the SMA analysis, the method was modified to base normalization on the control peak areas only, e.g., each probe area of Group B was normalized by dividing it by the mean of the control probe areas within Group B. Forty-eight goodquality 2:2 samples, each having an SD of the 21 control peak ratios at $<0.1$, were used as a reference group to construct the mean peak area of each probe (the very first synthetic control probe at 94 bp was not used for this extra quality check).

For each sample, the copy number of the $S M N$ genes was determined on the basis of the eight SMN probe ratios. If the ratios of SMN1-D01, SMN2-D01, SMN1-D07 and SMN2D07 were between 0.75 and 1.25 and the mean ratio of four SMN1 + 2 probes (SMN1/2-D03, SMN1/2-D04, SMN1/2D05, and SMN1/2-D06) was between 0.875 and 1.125 , the tested sample was interpreted to have 2:2. If the ratios of SMN1-D01 and SMN1-D07 were between 0.75 and 1.25, the ratios of SMN2-D01 and SMN2-D07 were between 0.25 and 0.75 and the mean ratio of the four SMN1 +2 probe was between 0.625 and 0.875 , the tested sample was interpreted to have 2:1. If the ratios of SMN1-D01, SMN2-D01, SMN1-D07, and SMN2-D07 were between 0.25 and 0.75 and the mean ratio of the four SMN1 +2 probes between 0.375 and 0.625 , the tested sample was interpreted to have 1:1 (an SMA carrier). Other combinations of SMN1 and SMN2 were deduced in a similar way. The combined copy number of SMN1 and SMN2 genes deduced from SMN1-D01, SMN2-D01, SMN1-D07, and SMN2-D07 was the same as that deduced from SMN1 + 2 probes, otherwise, the reaction should be repeated. For the carriers ascertained through DHPLC and/or MLPA, MLPA 


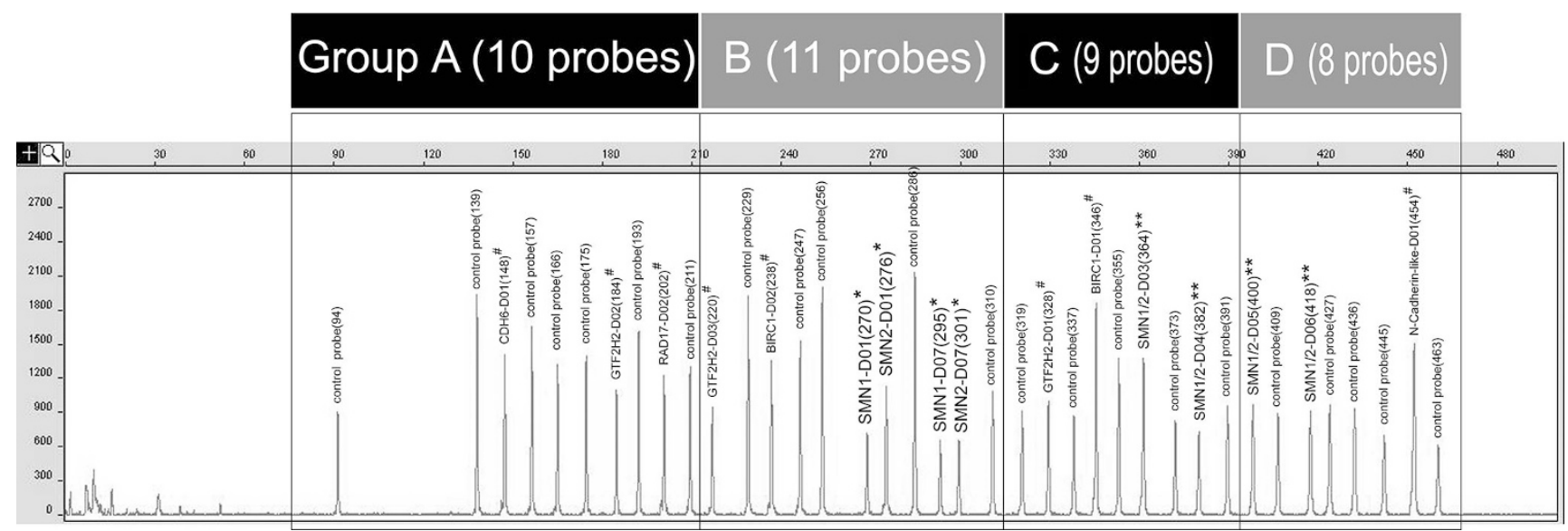

Figure 1. The electrophoreogram of the 38 probes. The numbers in parentheses indicate the length of the polymerase chain reaction (PCR) products in base pairs. The probes were divided into four groups, based on the size of the PCR products. The four probes indicated with an asterisk determined the respective copy numbers of exons 7 and 8 of $S M N 1$ and $S M N 2$, and the four with two asterisks determined the combined copy numbers of exons 1, 4, 6, and 8 of both $S M N$ genes. \# indicates 5q13 probes flanking the SMN1 or $S M N 2$. The other probes are located on chromosomes other than $5 \mathrm{q} 13$.

analysis was performed at least twice, and consistent results was obtained before the carrier status was established.

\section{RESULTS}

Figures 2 to 10 show the electrophoreograms of the various combinations of SMN1 and SMN2. For the 1:1 (Fig. 3) and 2:2 (Fig. 8) samples, the exon 7 peak in SMN2 was slightly higher than that in SMN1, whereas the exon 8 peaks were similar in SMN1 and SMN2. For the 2:0 samples (Fig. 6), a small signal was noted at the SMN2 exon 7 site and no signal was present at the SMN2 exon 8 site, whereas for the 0:3 samples (Fig. 2), as expected, no signals were present at both the SMN1 exon 7 and 8 sites. The presence of the SMN1 exon 7 sequence seemed to contribute to a portion of the exon 7 signal of SMN2.

By comparing the peak ratios of the tested sample against the reference group, we could obtain the copy numbers of the $S M N 1, S M N 2$, and neighboring genes. Although we set a \pm 0.25 ratio range to get the copy number for $S M N 1$ exons 7 and 8 and SMN2 exons 7 and 8 and set a \pm 0.125 mean ratio range to get the copy number for $S M N 1+S M N 2$, the actual ratio means for the 21 genotypes from Groups 1 and 2 were clear-cut with small SDs, usually $<10 \%$ of the mean (Table 1 ). For a very few cases having a slight discrepancy between a

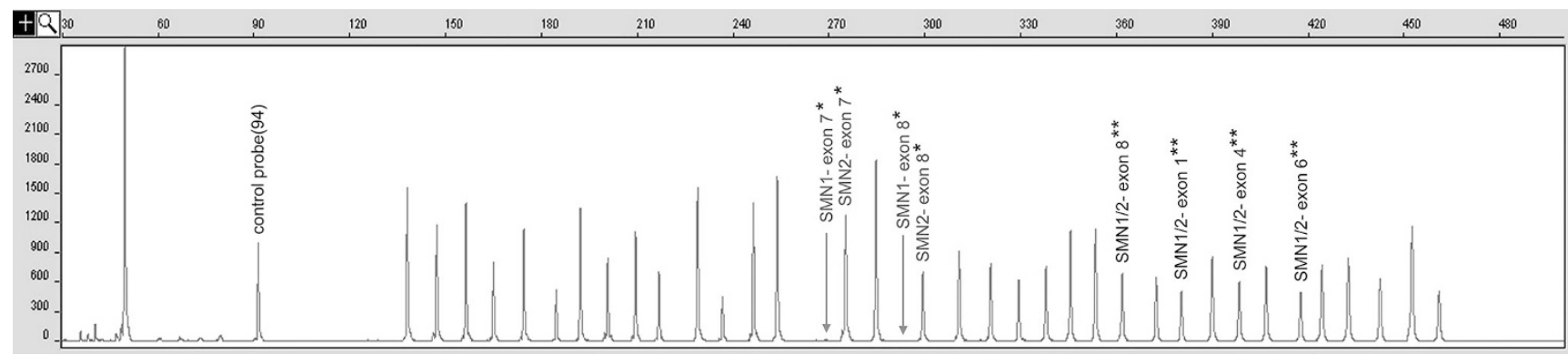

Figure 2. Electrophoreogram of $S M N 1: S M N 2=0: 3$. The arrows indicate the absence of the $S M N 1$ exon 7 and 8 peaks. The single and double asterisk codes are the same as in Figure 1 .

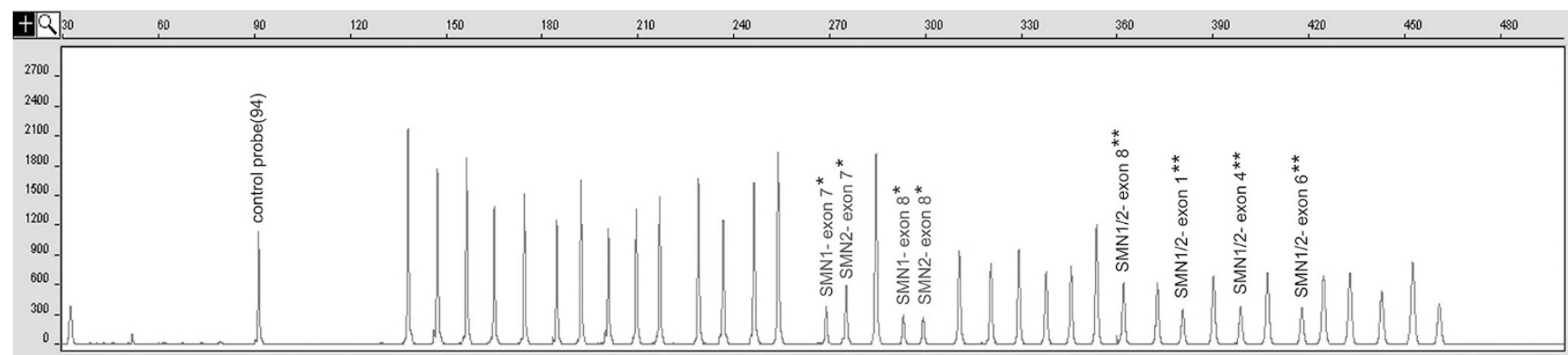

Figure 3. Electrophoreogram of $S M N 1: S M N 2=1: 1$. 


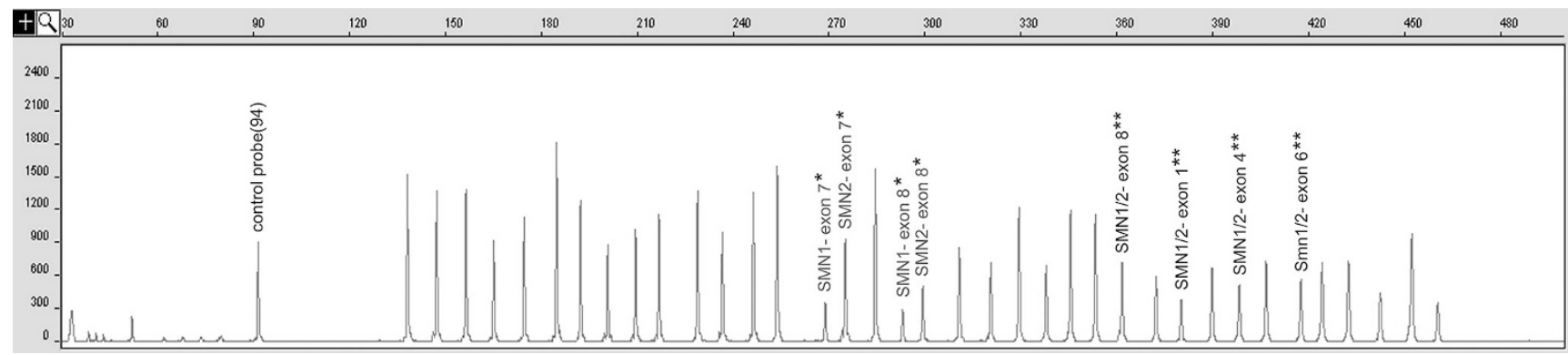

Figure 4. Electrophoreogram of $S M N 1: S M N 2=1: 2$.

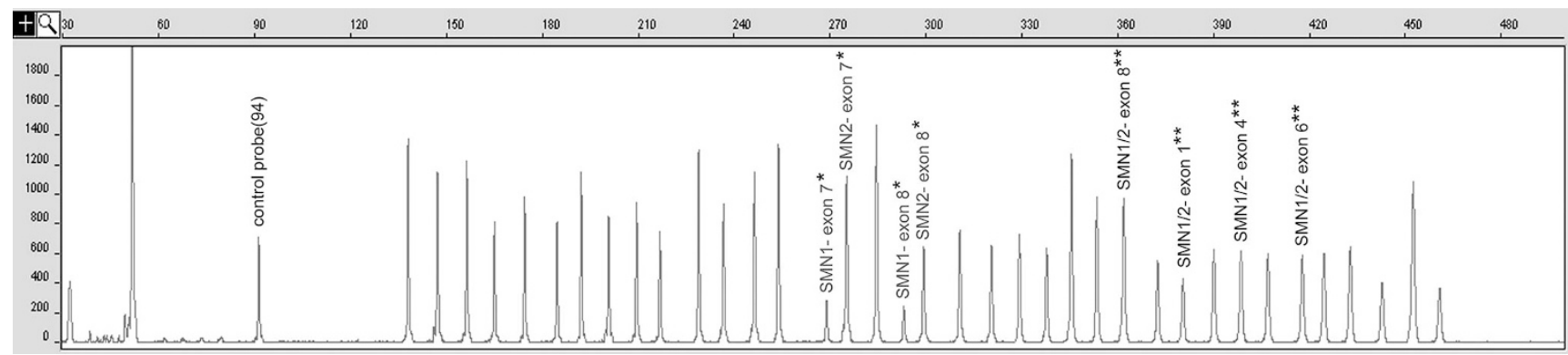

Figure 5. Electrophoreogram of SMN1:SMN2 = 1:3.

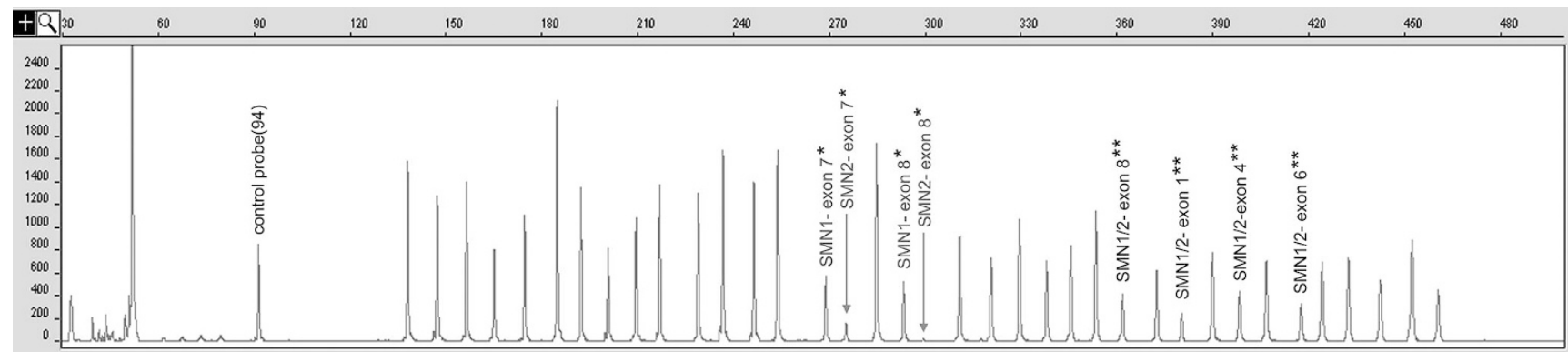

Figure 6. Electrophoreogram of $S M N 1: S M N 2=2: 0$. The arrows indicate the absence of $S M N 2$ exon 7 and 8 peaks. A small false band is present at the $S M N 2$ exon 7 site.

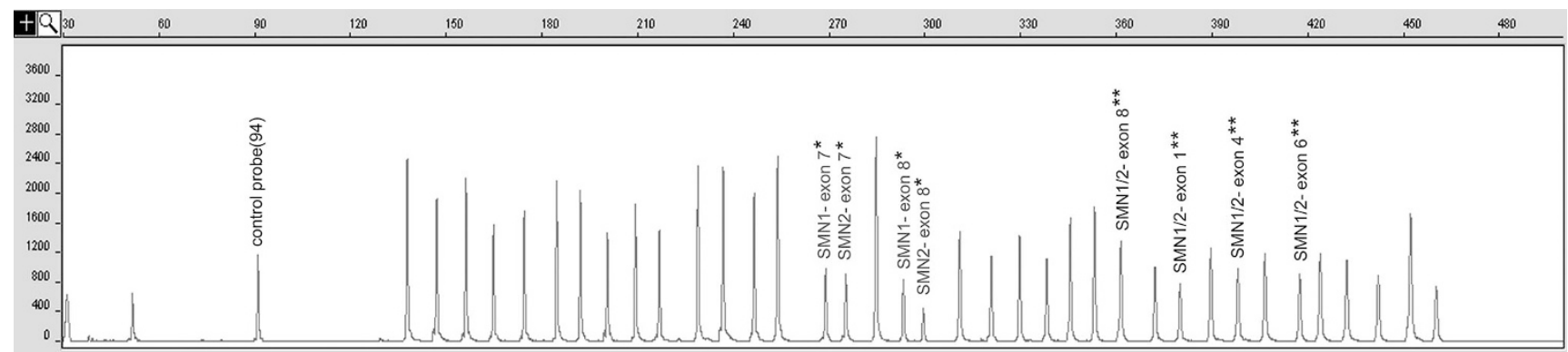

Figure 7. Electrophoreogram of $S M N 1: S M N 2=2: 1$.

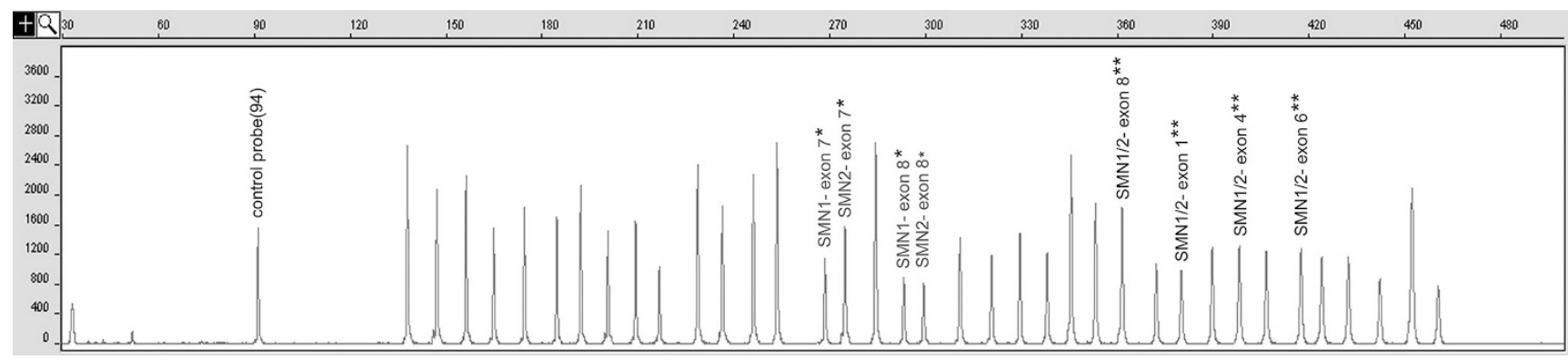

Figure 8. Electrophoreogram of $S M N 1: S M N 2=2: 2$. 


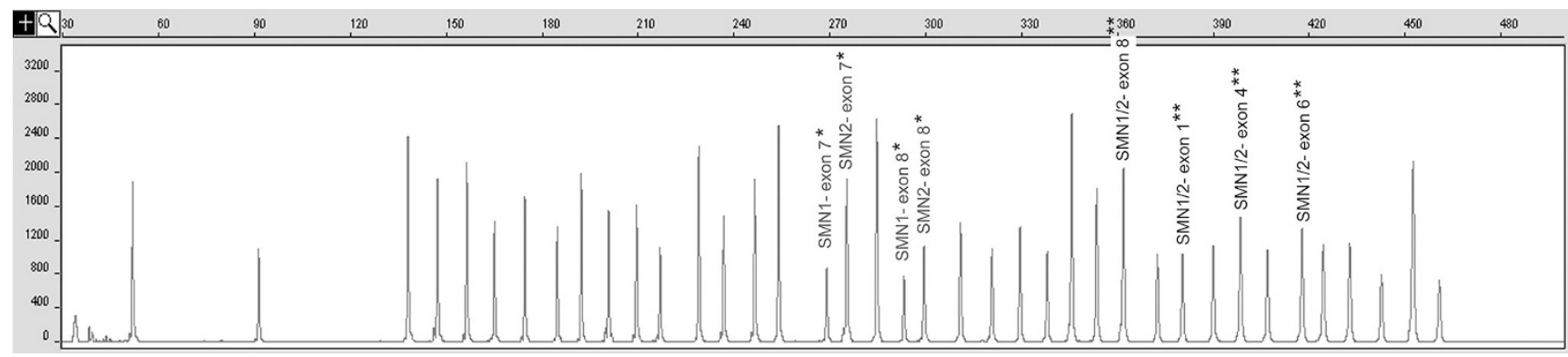

Figure 9. Electrophoreogram of $S M N 1: S M N 2=2: 3$.

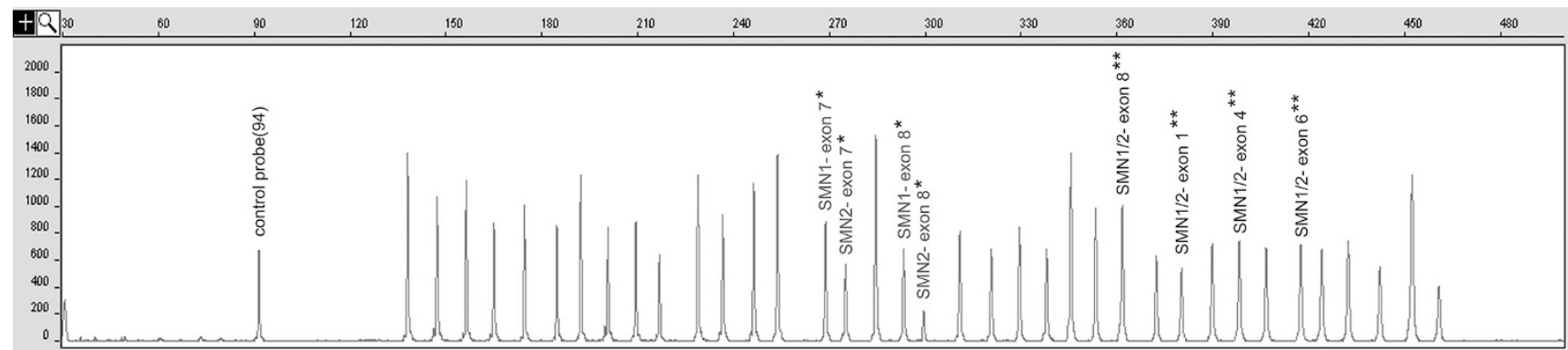

Figure 10. Electrophoreogram of $S M N 1: S M N 2=3: 1$.

Table 1

The mean $\pm \mathrm{SD}$ of the ratios of SMN exons in various combinations of SMN1 and SMN2 genes in subjects from Groups 1 and 2

\begin{tabular}{|c|c|c|c|c|c|c|c|}
\hline $\begin{array}{l}\text { Exon } 7 \\
\text { copies }\end{array}$ & $\begin{array}{c}\text { Exon } 8 \\
\text { copies }\end{array}$ & $\mathrm{n}$ & $\begin{array}{l}\text { Exon } 7 \\
\text { SMN1 }\end{array}$ & $\begin{array}{l}\text { Exon } 7 \\
\text { SMN2 }\end{array}$ & $\begin{array}{c}\text { Exon } 8 \\
\text { SMN1 }\end{array}$ & $\begin{array}{l}\text { Exon } 8 \\
\text { SMN2 }\end{array}$ & $S M N 1 / 2$ \\
\hline $0: 2$ & $0: 2$ & 7 & $0.00 \pm 0.00$ & $0.89 \pm 0.13$ & $0.00 \pm 0.00$ & $0.95 \pm 0.11$ & $0.53 \pm 0.06$ \\
\hline $0: 3$ & $0: 3$ & 6 & $0.00 \pm 0.00$ & $1.32 \pm 0.06$ & $0.00 \pm 0.00$ & $1.44 \pm 0.09$ & $0.77 \pm 0.05$ \\
\hline $0: 4$ & $0: 4$ & 5 & $0.00 \pm 0.00$ & $1.73 \pm 0.08$ & $0.00 \pm 0.00$ & $1.93 \pm 0.11$ & $1.04 \pm 0.04$ \\
\hline $1: 1$ & $1: 1$ & 9 & $0.53 \pm 0.06$ & $0.50 \pm 0.07$ & $0.52 \pm 0.06$ & $0.54 \pm 0.05$ & $0.52 \pm 0.03$ \\
\hline $1: 2$ & $1: 2$ & $29^{a}$ & $0.53 \pm 0.08$ & $0.94 \pm 0.12$ & $0.52 \pm 0.06$ & $1.02 \pm 0.08$ & $0.76 \pm 0.04$ \\
\hline $1: 3$ & $1: 3$ & $12^{b}$ & $0.53 \pm 0.04$ & $1.39 \pm 0.10$ & $0.49 \pm 0.04$ & $1.46 \pm 0.08$ & $1.00 \pm 0.06$ \\
\hline $1: 4$ & $1: 4$ & 1 & 0.56 & 1.74 & 0.50 & 1.88 & 1.20 \\
\hline $2: 0$ & $2: 0$ & 12 & $1.04 \pm 0.14$ & $0.10 \pm 0.08$ & $1.01 \pm 0.05$ & $0.00 \pm 0.00$ & $0.53 \pm 0.03$ \\
\hline $2: 1$ & $1: 2$ & $1^{c}$ & 1.12 & 0.67 & 0.56 & 1.00 & 0.72 \\
\hline $2: 1$ & $2: 1$ & 94 & $1.03 \pm 0.11$ & $0.57 \pm 0.07$ & $1.02 \pm 0.09$ & $0.53 \pm 0.05$ & $0.78 \pm 0.03$ \\
\hline $2: 1$ & $3: 0$ & $1^{c}$ & 1.17 & 0.42 & 1.58 & 0.00 & 0.76 \\
\hline $2: 2$ & $1: 3$ & $2^{c}$ & $1.03 \pm 0.02$ & $0.93 \pm 0.01$ & $0.53 \pm 0.00$ & $1.47 \pm 0.03$ & $1.00 \pm 0.00$ \\
\hline $2: 2$ & $2: 2$ & 164 & $1.03 \pm 0.10$ & $1.00 \pm 0.09$ & $1.01 \pm 0.07$ & $1.00 \pm 0.06$ & $1.01 \pm 0.03$ \\
\hline $2: 2$ & $3: 1$ & $4^{c}$ & $1.02 \pm 0.06$ & $1.03 \pm 0.07$ & $1.57 \pm 0.06$ & $0.57 \pm 0.02$ & $1.00 \pm 0.02$ \\
\hline $2: 3$ & $2: 3$ & 5 & $1.00 \pm 0.06$ & $1.36 \pm 0.05$ & $0.97 \pm 0.07$ & $1.43 \pm 0.09$ & $1.21 \pm 0.04$ \\
\hline $2: 4$ & $2: 4$ & 1 & 0.97 & 1.71 & 0.96 & 1.78 & 1.42 \\
\hline $3: 0$ & $3: 0$ & 3 & $1.45 \pm 0.05$ & $0.21 \pm 0.07$ & $1.55 \pm 0.08$ & $0.00 \pm 0.00$ & $0.77 \pm 0.03$ \\
\hline $3: 1$ & $3: 1$ & 11 & $1.53 \pm 0.12$ & $0.65 \pm 0.04$ & $1.52 \pm 0.12$ & $0.56 \pm 0.05$ & $1.02 \pm 0.04$ \\
\hline $3: 2$ & $3: 2$ & 3 & $1.45 \pm 0.14$ & $0.96 \pm 0.14$ & $1.50 \pm 0.14$ & $1.08 \pm 0.11$ & $1.23 \pm 0.00$ \\
\hline $4: 1$ & $4: 1$ & 2 & $2.15 \pm 0.03$ & $0.61 \pm 0.16$ & $2.03 \pm 0.06$ & $0.58 \pm 0.02$ & $1.17 \pm 0.05$ \\
\hline \multirow[t]{2}{*}{$4: 2$} & $4: 2$ & 1 & 2.03 & 1.16 & 1.94 & 1.03 & 1.46 \\
\hline & & 373 & & & & & \\
\hline
\end{tabular}

\footnotetext{
${ }^{a}$ Twenty-seven carriers from Group 2 and two carriers screened from Group 1.

${ }^{b}$ Eight carriers from Group 2 and four carriers screened from Group 1.
}

${ }^{c}$ Hybrid SMN gene. 
Table 2

The distribution of SMN1:SMN2 genes in 310 subjects in group 1

\begin{tabular}{lcc}
\hline SMN1:SMN2 ratio & No. of subjects & Ratio (\%) \\
\hline $1: 2$ & 2 & 0.65 \\
$1: 3$ & 4 & 1.29 \\
$2: 0$ & 12 & 3.87 \\
$2: 1$ & 94 & 30.32 \\
$2: 2$ & 164 & 52.90 \\
$2: 3$ & 5 & 1.61 \\
$2: 4$ & 1 & 0.32 \\
$3: 0$ & 3 & 0.97 \\
$3: 1$ & 11 & 3.55 \\
$3: 2$ & 3 & 0.97 \\
$4: 1$ & 2 & 0.65 \\
$4: 2$ & 1 & 0.32 \\
$2: 1 / 1: 2^{a}$ & 1 & 0.32 \\
$2: 1 / 3: 0^{a}$ & 1 & 0.32 \\
$2: 2 / 3: 1^{a}$ & 4 & 1.29 \\
$2: 2 / 1: 3^{a}$ & 2 & 0.65 \\
Total & 310 & \\
\hline & & \\
\hline & & \\
\hline
\end{tabular}

${ }^{a}$ Hybrid SMN genes, SMN1 exon 7:SMN2 exon 7/SMN1 exon 8:SMN2 exon 8.

found copy number ratio and the copy number for SMN1 + $S M N 2$, the copy number for the ratio was set to correspond to the SMN1 + SMN2 copy number.

Among the 16 different genotypes found in Group 1, the most common genotype was $2: 2(52.90 \%)$, followed by $2: 1$ $(30.32 \%)$. Six $(1.94 \%)$ subjects were found to be carriers of SMA. Eight subjects were noted to have one copy of a hybrid gene of SMN1 and SMN2 (Table 2). In Group 2, all 18 SMA patients had a total deletion of their SMN1 genes: seven patients with $0: 2$, six with $0: 3$, and five with $0: 4$. Among the 45 carriers, 9 had a 1:1, 27 had a 1:2, 8 had a 1:3, and 1 had a 1:4 genotype (Table 1). Eleven patients and 20 carriers from Groups 1 and 2 had a deletion extending to the SMN1 upstream genes of $B I R C 1$ and GTF2H2. All the seven 0:2 patients had at least one allele with the deletion extending beyond BIRC1 exon 5 (about $58 \mathrm{~kb}$ upstream to the SMN1 gene) and all the 0:4 patients did not have the deletion extending to the $B I R C 1$ exon 5 (Table 3 ). Among the seven patients with $0: 2$, six had Type I disease and one had Type II disease. Of the six patients with 0:3, five had Type II disease and one had Type I disease. All the five patients with 0:4 had Type III disease.

In Group 3, of the 20 subjects who had undergone DHPLC before to determine the status of their SMN genes, 14 had the same results with both DHPLC and MLPA; however, the other six had discrepant results. Three subjects who had been previously told they were carriers of SMA turned out to be noncarriers by MLPA (Table 4 ).
Table 3

Deletion extending to upstream SMN1 genes in 20 spinal muscular atrophy carriers and 18 patients

\begin{tabular}{|c|c|c|c|c|c|}
\hline Probe & SMN1-D01 & SMN1-D07 & BIRC1-D02 & GTF2H2-D02 & $\mathrm{n}$ \\
\hline Locus & Exon 7 & Exon 8 & Exon 5 & Exon 10 & \\
\hline $\begin{array}{l}\text { Distance } \\
\quad \text { to } S M N 1\end{array}$ & - & - & $58 \mathrm{~kb}$ & $95 \mathrm{~kb}$ & \\
\hline \multirow{2}{*}{$\begin{array}{l}\text { Carriers } \\
\qquad(n=51)\end{array}$} & $\mathrm{N}^{a} / \mathrm{del}^{b}$ & N/del & N/del & $\mathrm{N} / \mathrm{del}$ & 14 \\
\hline & N/del & N/del & N/del & $\mathrm{N} / \mathrm{N}$ & \\
\hline \multirow{4}{*}{$\begin{array}{c}0: 2 \text { patients } \\
\quad(n=7)\end{array}$} & $\mathrm{del} / \mathrm{del}$ & $\mathrm{del} / \mathrm{del}$ & $\mathrm{del} / \mathrm{del}$ & $\mathrm{del} / \mathrm{del}$ & \\
\hline & $\mathrm{del} / \mathrm{del}$ & $\mathrm{del} / \mathrm{del}$ & N/del & $\mathrm{N} / \mathrm{del}$ & \\
\hline & $\mathrm{del} / \mathrm{del}$ & $\mathrm{del} / \mathrm{del}$ & $\mathrm{del} / \mathrm{del}$ & $\mathrm{N} / \mathrm{N}$ & \\
\hline & del/del & $\mathrm{del} / \mathrm{del}$ & N/del & $\mathrm{N} / \mathrm{N}$ & \\
\hline \multirow{3}{*}{$\begin{array}{c}0: 3 \text { patients } \\
\quad(n=6)\end{array}$} & $\mathrm{del} / \mathrm{del}$ & $\mathrm{del} / \mathrm{del}$ & N/del & $\mathrm{N} / \mathrm{del}$ & \\
\hline & $\mathrm{del} / \mathrm{del}$ & $\mathrm{del} / \mathrm{del}$ & N/del & $\mathrm{N} / \mathrm{N}$ & \\
\hline & $\mathrm{del} / \mathrm{del}$ & $\mathrm{del} / \mathrm{del}$ & $\mathrm{N} / \mathrm{N}$ & $\mathrm{N} / \mathrm{N}$ & \\
\hline $\begin{array}{c}0: 4 \text { patients } \\
\quad(n=5)\end{array}$ & $\mathrm{del} / \mathrm{del}$ & $\mathrm{del} / \mathrm{del}$ & $\mathrm{N} / \mathrm{N}$ & $\mathrm{N} / \mathrm{N}$ & \\
\hline
\end{tabular}

${ }^{a}$ No deletion detected.

${ }^{b}$ Deletion detected.

Table 4

Discrepant results of SMN gene copy number by DHPLC and MLPA

\begin{tabular}{lccc}
\hline Subject & DHPLC & MLPA & Interpretation \\
\hline A & $1: 3$ & $2: 4$ & $\begin{array}{c}\text { SMA carrier by DHPLC, } \\
\text { noncarrier by MLPA } \\
\text { B }\end{array}$ \\
C & $1: 2$ & $2: 3$ & $\begin{array}{c}\text { SMA carrier by DHPLC, } \\
\text { noncarrier by MLPA } \\
\text { SMA carrier, different SMN2 copy } \\
\text { number }\end{array}$ \\
D & $1: 3$ & $1: 4$ & $\begin{array}{c}\text { Noncarrier, different SMN2 copy } \\
\text { number }\end{array}$ \\
E & $2: 1$ & $2: 2$ & $\begin{array}{c}\text { SMA carrier, different SMN2 copy } \\
\text { number }\end{array}$ \\
F & $1: 3$ & $1: 2$ & $\begin{array}{c}\text { SMA carrier by DHPLC, } \\
\text { noncarrier by MLPA }\end{array}$ \\
\hline
\end{tabular}

DHPLC, denatured high-performance liquid chromatography; MLPA, multiplex ligation-dependent probe amplification; SMA, spinal muscular atrophy.

Sixteen genotypes were found in Group 1 subjects (Table 1 ), and five genotypes (0:2, 0:3, 0:4, 1:1, and 1:4) were found in Group 2, yielding a total of 21 genotypes detected in this series.

\section{DISCUSSION}

Copy number analysis of the SMN1 gene is important in carrier screening and prenatal diagnosis. ${ }^{1,2,9,26,27}$ Many methods have been developed for this purpose. ${ }^{7-9,12,13}$ Most of them recognize the only nucleotide difference in exon 7 between SMN1 and SMN2. SSCP is a simple method, but its sensitivity is not high. ${ }^{14}$ PCR-RFLP is now commonly used in clinical 
practice. ${ }^{7}$ Although these methods can detect most cases of homozygous deletion of $S M N 1$ genes, they cannot always offer a clear-cut distinction between a deletion SMA carrier and a noncarrier. Single-tube MLPA as reported here uses a single pair of PCR primers to simultaneously amplify up to 38 different DNA sequences. ${ }^{15}$ This method detected the unique sequences of exons 7 and 8 of $S M N 1$, exons 7 and 8 of $S M N 2$, and the common sequences of exons $1,4,6$, and 8 of both $S M N 1$ and $S M N 2$ in a single reaction. In addition, nine loci in the flanking regions of SMN genes and 22 genomic control sequences were studied in the same tube. Therefore, many internal and external controls were analyzed under the same conditions for better quality control and data confirmation.

The performance of genomic controls could indicate the quality of extracted DNA, the quantity of the input DNA, and the efficiency of DNA ligation and amplification. A good-quality reaction had an $\mathrm{SD}<0.1$ of the peak ratios of the 21 control probes. Data not from a good-quality reaction should be interpreted with extra caution or the whole process should be repeated, starting from blood sample collection, to obtain a correct classification. The copy number of SMN1 and SMN2 determined from the unique sequences of exons 7 and 8 should be compatible with that determined from the common sequences of exons 1, 4, 6, and 8 of both SMN1 and SMN2. In addition, we were able to compare the copy number of exon 7 of SMN1 and SMN2 with that of exon 8 of SMN1 and SMN2. Eight subjects in Group 1 were thus interpreted to have a hybrid SMN gene (Table 1).

Loci flanking the $S M N$ genes could confirm deletion in the $S M N$ genes and help delineate the extent of the deletion. Patients with a more extensive deletion involving BIRC1 and GTF2H2 seemed to have a more severe phenotype. Twenty of the 51 SMA carriers had the deletion extending at least $58 \mathrm{~kb}$ upstream of the SMN1 gene (Table 3). A higher copy number of the SMN2 also had an ameliorating effect on the severity of SMA.5,6

Three of the 19 subjects in Group 3 (Table 4) who had been told they were SMA carriers using DHPLC turned out to be noncarriers by MLPA. Two subjects had 2:3 and one had 2:4 $S M N$ genes by MLPA. The presence of five copies and six copies of $S M N$ genes were confirmed by copy number analysis of the SMN1/SMN2 probe study. MLPA analysis in these subjects was performed at least twice, and consistent results were obtained. Misclassification of a noncarrier as an SMA carrier may cause undue anxiety for the subject. In another two subjects, the copy number of SMN2 by DHPLC was different from that by MLPA (Table 4). Although the determination of the SMN2 copy number is less important, this misclassification may be the tip of an iceberg. Currently all the DHPLC screenings for the SMN genes in Taiwan have been performed according to the protocol reported by Su et al. ${ }^{13}$ They use a two-step method: (1) The first step of heteroduplex analysis only studies the ratio of SMN1/ SMN2 to detect the SMA carriers with a ratio other than 1. (2) The second step is a multiplex competitive PCR to calculate the total copy numbers of SMN1 + SMN2 to distinguish the SMA carriers with 1:1 from the normal subjects with 2:2. In the second step, they use two control probes, CYBB (OMIM\#306400, X-linked) and KRIT1 (OMIN\#604214, chromosome 7q), to control for the total copy number of SMN genes. All the DHPLC results in this report seemed to have been screened with only the first-step method; therefore, 1:2 and 2:3 would have been difficult to distinguish. We believe that if the second-step procedure had been undertaken, these misclassifications would have been avoided. With MLPA, many control probes, both in the SMN region and other chromosomes, were used for total copy number reference for $S M N$ gene or for system quality control. Copy number analysis of $S M N$ genes with MLPA would be better and more efficient than the two-step DHPLC. ${ }^{13}$

Detailed analysis to pinpoint the underlying causes is needed to avert misclassification of the copy number of the SMN1 gene. Although DHPLC has been successfully used in SMA carrier screening, the techniques may go wrong in inexperienced hands. The discrepancies in Group 3 may require an independent assay to clarify the status of $S M N$ genes; however, we believe that it was less likely that MLPA results were wrong.

About $5 \%$ of the diseased SMA alleles are caused by point mutations in the coding or splicing junction region of the SMN1 gene. ${ }^{5}$ MLPA SMN copy number analysis cannot detect these intragenic mutations. Strategies to identify these subtle mutations are needed for a complete detection of all SMA defects. ${ }^{28}$ As demonstrated in other diseases, MLPA is a simple and effective method to determine the copy number of SMN1 and SMN2 and can play an important role in the diagnosis of patients and the mass screening of SMA carriers.

\section{References}

1. Cusin V, Clermont O, Gerard B, Chantereau D, et al. Prevalence of SMN1 deletion and duplication in carrier and normal populations: implication for genetic counseling. J Med Genet 2003;40:e39.

2. Feldkotter M, Schwarzer V, Wirth R, Wienker TF, et al.Quantitative analyses of SMN1 and SMN2 based on real-time LightCycler PCR: fast and highly reliable carrier testing and prediction of severity of spinal muscular atrophy. Am J Hum Genet 2002;70:358-368

3. Ogino S, Wilson RB. Genetic testing and risk assessment for spinal muscular atrophy (SMA). Hum Genet 2002;111:477-500.

4. Zerres K, Rudnik-Schoneborn S. Natural history in proximal spinal muscular atrophy. Clinical analysis of 445 patients and suggestions for a modification of existing classifications. Arch Neurol 1995;52:518-523.

5. Ogino S, Wilson RB. Spinal muscular atrophy: molecular genetics and diagnostics. Expert Rev Mol Diagn 2004;4:15-29.

6. Frugier T, Nicole S, Cifuentes-Diaz C, Melki J. The molecular bases of spinal muscular atrophy. Curr Opin Genet Dev 2002;12:294-298.

7. Wirth B, Herz M, Wetter A, Moskau S, et al. Quantitative analysis of survival motor neuron copies: identification of subtle SMN1 mutations in patients with spinal muscular atrophy, genotype-phenotype correlation, and implications for genetic counseling. Am J Hum Genet 1999;64:1340-1356.

8. Sutomo R, Akutsu T, Takeshima Y, Nishio H, et al. Rapid SMN1 deletion test using DHPLC to screen patients with spinal muscular atrophy. Am J Med Genet 2002;113: 225-226.

9. Mazzei R, Conforti FL, Muglia M, Sprovieri T, et al. A simple method for diagnosis of autosomal recessive spinal muscular atrophy by denaturing high-performance liquid chromatography. J Child Neurol 2003;18:269-271.

10. Chen KL, Wang YL, Rennert H, Joshi I, et al. Duplications and de novo deletions of the SMNt gene demonstrated by fluorescence-based carrier testing for spinal muscular atrophy. Am J Med Genet 1999;85:463-469.

11. Tsai CH, Jong YJ, Hu CJ, Chen CM, et al. Molecular analysis of SMN, NAIP, and P44 genes of SMA patients and their families. J Neurol Sci 2001;190:35-40.

12. Cusco I, Barcelo MJ, Baiget M, Tizzano F. Implementation of SMA carrier testing in genetic laboratories: comparison of two methods for quantifying the SMN1 gene. Hum Mutat 2002;20:452-459. 
13. Su YN, Hung CC, Li H, Lee CN, et al. Quantitative analysis of SMN1 and SMN2 genes based on DHPLC: a highly efficient and reliable carrier-screening test. Hum Mutat 2005;25:460-467.

14. Lefebvre S, Burglen L, Reboullet S, Clermont O, et al. Identification and characterization of a spinal muscular atrophy-determining gene. Cell 1995;80:155-165.

15. Schouten JP, McElguun CJ, Waaijer R, Zwijnenburg D, et al. Relative quantification of 40 nucleic acid sequences by multiplex ligation-dependent probe amplification. Nucleic Acids Res 2002;30:e57.

16. Lai KKS, Lo IFM, Tong TMF, Cheng LYL, et al. Detecting exon deletions and duplications of the DMD gene using Multiplex ligation-dependent probe amplification (MLPA). Clin Biochem 2006;39:367-372.

17. Gerdes T, Kirchhoff M, Lind AM, Laesen GV, et al. Computer-assisted prenatal aneuploidy screening for chromosome 13, 18, 21, X and Y based on multiplex ligationdependent probe amplification (MLPA). Eur J Hum Genet 2005;13:171-175.

18. Slater HR, Bruno DL, Ren H, Pertile M, et al. Rapid, high throughput prenatal detection of aneuploidy using a novel quantitative method (MLPA). J Med Genet 2003;40:907-912.

19. Hochstenbach R, Meijer Jvan de Brug J, et al. Rapid detection of chromosomal aneuploidies in uncultured amniocytes by multiplex ligation-dependent probe amplification. Prenat Diagn 2005;25:1032-1039.

20. Montagna M, Palma MD, Menin C, Agata S, et al. Genomic rearrangements account for more than one-third of the BRCA1 mutations in northern Italian breast/ovarian cancer families. Hum Mol Genet 2003;12:1055-1061.
21. Taylor CF, Charlton RS, Burn J, Sheridan E, et al. Genomic deletions in MSH2 or MLH1 are a frequent cause of hereditary non-polyposis colorectal cancer: identification of novel and recurrent deletions by MLPA. Hum Mutat 2003;22:428433.

22. Fernandez L, Lapunzina P, Arjona D, Lopez PI, et al. Comparative study of three diagnostic approaches (FISH, STRs and MLPA) in 30 patients with 22q11.2 deletion syndrome. Clin Genet 2005;68:373-378.

23. Tomaszewicz K, Kang P, Wu BL. Detection of homozygous and heterozygous SMN deletion of spinal muscular atrophy in a single assay with multiplex ligation-dependent probe amplification. Beijing Da Xue Xue Bao 2005;37:55-57.

24. Scarciolla O, Stuppia L, De Angelis MV, Murru S, et al. Spinal muscular atrophy genotyping by gene dosage using multiple ligation-dependent probe amplification. Neurogenetics 2006; 7:269-276.

25. Gerdes T, Kirchhoff M, Bryndorf T. Automatic analysis of multiplex ligation-dependent probe amplification products (exemplified by a commercial kit for prenatal aneuploidy detection). Electrophoresis 2005;26:4327-4332.

26. Lin SP, Chang JG, Jong YJ, Yang TY, et al. Prenatal prediction of spinal muscular atrophy in Chinese. Prenat Diagn 1999;19:657-661.

27. Kesari A, Rennert H, Leonard DGB, Phadke S, et al. Prenatal diagnosis of spinal muscular atrophy: Indian scenario. Prenat Diagn 2005;25:641-644.

28. Clermont O, Burlet P, Benit P, Chanterau D, et al. Molecular analysis of SMA patients without homozygous SMN1 deletions using a new strategy for identification of SMN1 subtle mutations. Hum Mutat 2004;24:417-427. 